\title{
3D SIMULATION OF WAVE INTERACTION WITH PERMEABLE STRUCTURES
}

\author{
P.R. Wellens ${ }^{1}$, M.J.A Borsboom ${ }^{1}$ and M.R.A van Gent ${ }^{1}$
}

\begin{abstract}
COMFLOW is a general 3D free-surface flow solver. The main objective in this paper is to extend the solver with a permeable flow model to simulate wave interaction with rubble-mound breakwaters. The extended Navier-Stokes equations for permeable flow are presented and we show the discretization of these equations as they are implemented in COMFLOW. An analytical solution for the reflection coefficient of a permeable structure is derived and the numerical model is compared to the solution. In addition, a validation study has been performed, in which we compare the numerical results with an experiment. In the experiment, pressures and surface elevations are measured inside a permeable structure. The measurements are represented well by the simulation results. At the end, a 3D application of the model is shown.
\end{abstract}

Keywords: VOF; 3D Navier-Stokes, permeability; rubble-mound breakwater; $3 D$ simulations

\section{INTRODUCTION}

Designs of coastal structures are often evaluated by means of experiments in a model basin. During an experiment we assess the damage to a model structure due to a representative storm, and determine whether the damage is above or below a certain threshold. Besides damage levels, parameters such as wave run-up, overtopping and transmission, resulting from the interaction between waves and the coastal structure, can be measured, depending on the scope of the experiment.

Numerical simulations can be performed in support of these experiments. One advantage of numerical simulation is that output of the solution variables may straightforwardly be obtained from the entire domain, whereas it is common in experiments that only local information with pin-point measurements is obtained. Data of a global nature may prove advantageous in the explanation of phenomena observed in the experiments.

There are not many models for the detailed simulation of waves near structures. Phenomena such as wave run-up or wave impacts on the slopes of a structure, or the overtopping of its crest, require a specific type of modeling. ComFLOW (e.g. Kleefsman et al., 2005) is a 3D Volume of Fluid (VOF) model designed specifically for this type of event, see Fig.1. For applications with permeable coastal structures, it has its origins in the 2D VOF model presented by Van Gent $e$ al. (1994). A similar approach to the latter with respect to coastal structures was proposed by Liu et al. (1999). COMFLOW is in active development and well validated in recent years, for, predominantly, offshore applications. A coastal application is presented in Wenneker et al. (2010).

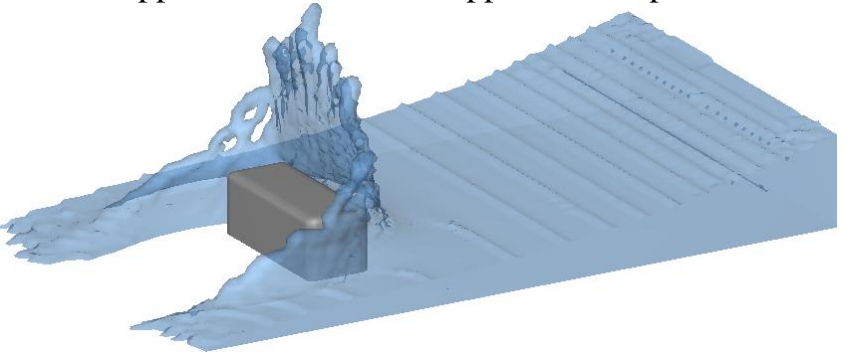

Figure 1. Dambreak simulation in ComFLOW: impact with a structure in the trajectory of the flow.

ComFLOW is based on the 3D Navier-Stokes equations in both water and air. It employs a symmetry-preserving discretization of these equations on a Cartesian grid (Verstappen and Veldman, 2003) and the free surface is advected by means of the Volume of Fluid method, which has been extended with a local height function to prevent droplets from disconnecting from the free surface. A cut-cell method is used to include arbitrarily shaped geometries in the computational domain (Kleefsman et al., 2005).

The emphasis in the design of the numerical model has been on momentum-driven flows in the presence of a free surface, meaning that pressures as a result of wave impacts on a structure are resolved more accurately than vortices shedding from, for instance, a cylinder in a uniform flow.

\footnotetext{
${ }^{1}$ Deltares, P.O. Box 177, 2600 MH Delft, The Netherlands; peter.wellens@deltares.nl
} 
Coastal structures are often composed of rubble-mound material. Damage to the structure, runup and wave overtopping are strongly influenced by the permeability of the structure. For phenomena such as wave overtopping and wave transmission it is even required that the wave dynamics are computed throughout the structure.

For the detailed simulation of wave phenomena near coastal structures, therefore, it is important to include the effects of flow through permeable media.

\section{GOVERNING EQUATIONS}

For flow through permeable media, the Navier-Stokes equations need to account for the porosity of the medium. With COMFLOW, we will not compute the flow through the pores themselves, i.e. the grid resolution in our simulations will be much coarser than the pores of the rock material that we wish to model. Therefore, a volume-averaged method is adopted, in which we assume that the properties of the permeable structure, such as the porosity, are homogenous throughout (part of) the structure.

Besides porosity, we also need to account for the viscous interaction of the flow with the rocks in the permeable structure. We cannot represent all the (turbulent) boundary layers around the individual stones and will therefore model the viscous interaction with an additional volume averaged friction force in the Navier-Stokes equations that depends on the flow velocity. A similar approach is adopted in Darcy's Law, which was adapted by Forchheimer to account for turbulent flows. The friction force is as follows:

$$
R(u)=(\hat{a}+\hat{b}|u|) u
$$

in which $\hat{a}$ is a Darcy-type coefficient, $\hat{b}$ is a Forchheimer-type coefficient and $u$ is the actual flow velocity in the permeable structure and not the bulk velocity. Friction force (1) is part of the extended Navier-Stokes equations for permeable flow. The extended continuity equation for permeable flow is:

$$
\nabla \cdot(\varepsilon u)=0
$$

in which $\varepsilon$ is the porosity. We assume constant viscosity and an incompressible fluid. Then the momentum equation reads:

$$
\varepsilon \frac{\partial u}{\partial t}+\nabla \cdot(u \varepsilon u)=v \nabla \cdot(\varepsilon \nabla u)-\frac{\varepsilon}{\rho} \nabla p-\varepsilon R(u)+\varepsilon F
$$

Here, $v$ is the viscosity of the fluid, $\rho$ is the density of the fluid, $p$ is the pressure and $F$ is an external force vector (such as, for instance, gravity).

\section{DISCRETIZATION}

Eqs. (2) and (3) require discretization for the implementation in CoMFLOW. In this section, the discretization of the individual terms in the Navier-Stokes equation will be discussed. These terms will be combined later in the paragraph concerning the time discretization. In CoMFLOW, a finite volume discretization has been adopted. To that end, we will now present the weak form of the Navier-Stokes equations. The continuity equation:

$$
\int_{\Gamma}(\varepsilon u) \cdot n \partial V
$$

Here, $n$ is the normal vector to the boundary of the control volume $\Gamma$. The weak formulation of the momentum equation becomes:

$$
\begin{aligned}
\varepsilon \int_{\Omega} \frac{\partial u}{\partial t} d V+\int_{\Gamma} u(u \varepsilon \cdot n) d S= & \\
& \quad \int_{\Gamma}(\varepsilon \nabla u) \cdot n d S-\frac{\varepsilon}{\rho} \int_{\Gamma} p n d S-\varepsilon \int_{\Omega} R(u) d V+\varepsilon \int_{\Omega} F d V
\end{aligned}
$$




\section{Space discretization of the continuity equation}

Fig. 2 shows a control volume for the continuity equation. The control volume corresponds to a grid cell on which the flow variables are defined. Note that the variables are staggered; velocities are defined at the cell faces, the pressure is defined in the cell centre (not shown in Fig. 2).

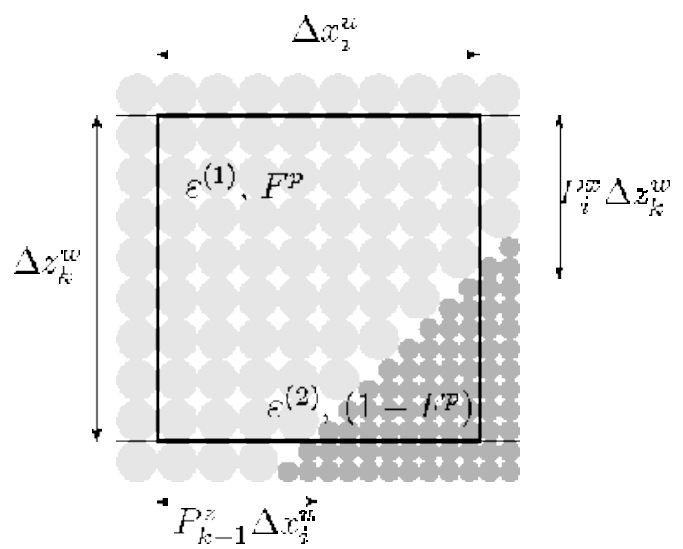

Figure 2. Control volume for the continuity equation.

The control volume in Fig. 2 is divided in two regions with different porosity. The grid sizes $\Delta x_{i}^{u}$ and $\Delta z_{k}^{w}$ are scaled by means of the dimensionless coefficients $P_{i}^{x}$ and $P_{k}^{z}$ to represent the outer dimensions of the two regions within the cell. The volumes of the regions are given by $F^{p} \Delta x_{i}^{u} \Delta z_{k}^{w}$ and $\left(1-F^{p}\right) \Delta x_{i}^{u} \Delta z_{k}^{w}$. Note, however, that the volume of the cell that is open for fluid, in which we account for the porosity, is equal to:

$$
F^{b}=\left(\varepsilon^{(1)} F^{p}+\varepsilon^{(2)}\left(1-F^{p}\right)\right) \Delta x_{i}^{u} \Delta z_{k}^{w}
$$

We will determine the flow through the pores of the cell face indicated by $i$ :

$$
\Phi_{i}=u_{i} A_{i}^{x} \Delta z_{k}^{w}
$$

in which $\Phi_{\mathrm{i}}$ represents the flux through the cell face with index $i$ and:

$$
A_{i}^{x}=\varepsilon^{(1)} P_{i}^{x}+\varepsilon^{(2)}\left(1-P_{i}^{x}\right)
$$

is what we call an aperture. It is the area of the cell face that is open to fluid. The discrete equivalent of the continuity equation in Eq. (4) is as follows:

$$
\Phi_{i}-\Phi_{i-1}+\Phi_{k}-\Phi_{k-1}=0
$$

And after substitution of all fluxes $\Phi$ around the circumference of the control volume, the discrete continuity equation reads:

$$
\left(u_{i} A_{i}^{x}-u_{i-1} A_{i-1}^{x}\right) \Delta z_{k}^{w}+\left(w_{k} A_{k}^{z}-w_{k-1} A_{k-1}^{z}\right) \Delta x_{i}^{u}=0
$$

\section{Space discretization of the momentum equation}

\section{Time derivative}

In Fig. 3, a control volume for the momentum equation is shown. The control volume is defined in between two consecutive grid cells. In the momentum equation, we require the size of the control volume. It is determined as the weighted average of the grid cell volumes, such as in Fig. 2, on either side. First, we determine the volume of the pores in cell with index $i$ : 


$$
F_{i}^{b}=\varepsilon^{(1)} F_{i}^{p}+\varepsilon^{(2)}\left(1-F_{i}^{p}\right)
$$

Then we determine the volume of the open region in the cell with index $i+1$ :

$$
F_{i+1}^{b}=\varepsilon^{(1)} F_{i+1}^{p}+\varepsilon^{(2)}\left(1-F_{i+1}^{p}\right)
$$

And finally, we determine the weighted average of the two:

$$
F_{i}^{u}=\frac{F_{i}^{b} \Delta x_{i}^{u}+F_{i+1}^{b} \Delta x_{i+1}^{u}}{\Delta x_{i}^{u}+\Delta x_{i+1}^{u}}
$$

The space discretization of the time derivative of the momentum equation then becomes:

$$
\varepsilon \int_{\Omega} \frac{\partial u}{\partial t} d V \approx F_{i}^{u} \Delta x_{i}^{p} \Delta z_{k}^{w} \frac{\partial u}{\partial t}
$$

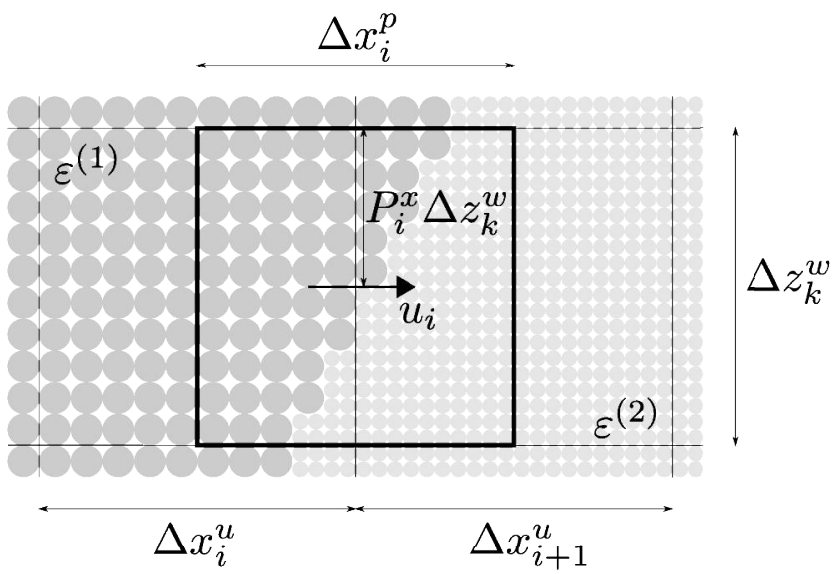

Figure 3. Control volume for the momentum equation.

\section{Convective term}

The Finite Volume discretization of the convective term is equal to the combination of fluxes over the boundary of the control volume:

$$
\int_{\Gamma} u(u \varepsilon \cdot n) d S \approx \Phi_{i+\frac{1}{2}}-\Phi_{i-\frac{1}{2}}+\Phi_{k+\frac{1}{2}}-\Phi_{k-\frac{1}{2}}
$$

in which the fluxes through the right and the top control volume boundary are:

$$
\begin{aligned}
& \Phi_{i+\frac{1}{2}}= \frac{1}{2}\left(u_{i, k} A_{i}^{x}+u_{i+1, k} A_{i+1}^{x}\right) \Delta z_{k}^{w} \frac{1}{2}\left(u_{i, k}+u_{i+1, k}\right)+ \\
& \frac{1}{2} \xi\left|u_{i, k} A_{i, k}^{x}+u_{i+1, k} A_{i+1, k}^{x}\right| \Delta z_{k}^{w} \frac{1}{2}\left(u_{i, k}-u_{i+1, k}\right) \\
& \Phi_{k+\frac{1}{2}}= \frac{1}{2}\left(w_{i, k} A_{i, k}^{z}+w_{i+1,1} A_{i+1, k}^{z}\right) \Delta x_{i}^{u} \frac{1}{2}\left(u_{i, k}+u_{i, k+1}\right)+ \\
& \frac{1}{2} \xi\left|w_{i, k} A_{i, k}^{z}+w_{i+1, k} A_{i+1, k}^{z}\right| \Delta x_{i}^{u} \frac{1}{2}\left(u_{i, k}-u_{i, k+1}\right)
\end{aligned}
$$

Note in (16) that the parameter $\xi$ determines the amount of upwind that is specified. The apertures $A$ in Eq. (16) can be determined from Fig. 3. As an example we will provide:

$$
A_{i}^{x}=\varepsilon^{(1)} P_{i}^{x}+\varepsilon^{(2)}\left(1-P_{i}^{x}\right)
$$




\section{Viscous term}

The discretization of the viscous term in this paper is relatively inaccurate compared to the other terms discussed here because it accounts for the porosity in a simplified manner. There are two reasons why we may assume that this discretization does not influence the results to a great extent. With COMFLOW we are primarily interested in wave loads as a result of steep waves; the type of flow in these waves is dominated by convection. In addition, inside permeable structures, the friction force term is by far dominant over the viscous term. For the discretization of the viscous term, we revert to the volume integral:

$$
v \int_{\Omega} \nabla \cdot \nabla u d V
$$

The divergence of the gradient of $u$ is approximated as follows, see Fig. 3:

$$
\nabla \cdot \nabla u \approx \Psi=\frac{1}{\Delta x_{i}^{p}}\left(\frac{u_{i+1, k}-u_{i, k}}{\Delta x_{i+1}^{u}}-\frac{u_{i, k}-u_{i-1, k}}{\Delta x_{i}^{u}}\right)+\frac{1}{\Delta z_{k}^{w}}\left(\frac{u_{i, k+1}-u_{i, k}}{\Delta z_{k}^{p}}-\frac{u_{i, k}-u_{i, k-1}}{\Delta z_{k-1}^{p}}\right)
$$

And Eq. (19) is multiplied by the volume of the momentum cell (see Eq. (13)) to obtain the full discretization of the viscous term:

$$
v \int_{\Omega} \nabla \cdot \nabla u d V \approx v F_{i}^{u} \Delta x_{i}^{p} \Delta z_{k}^{w} \Psi
$$

\section{Pressure and force term}

For the pressure term we adopt the following discretization, see Fig. 3:

$$
\frac{\varepsilon}{\rho} \int_{\Omega} p n d S \approx \frac{1}{\rho}\left[\left(p_{i+1}-p_{i}\right) \varepsilon^{(1)} P_{i}^{x}+\left(p_{i+1}-p_{i}\right) \varepsilon^{(2)}\left(1-P_{i}^{x}\right)\right] \Delta z_{k}^{w}(21)
$$

For the force term, a discretization is adopted in such a way that in hydrostatic circumstances the discrete form of $1 / \rho \nabla p=F$ is satisfied. The discretization of the force term in $x$-direction then becomes:

$$
\int_{\Omega} F_{x} d V \approx\left[F_{x} \varepsilon^{(1)} P_{i}^{x}+F_{x} \varepsilon^{(2)}\left(1-P_{i}^{x}\right)\right] \Delta x_{i}^{p} \Delta z_{k}^{w}
$$

\section{Friction force term}

For the integration of the friction force term over the control volume, we adopt a weighted average method. The friction force in the control volume with index $i$ (see Fig. 3) is:

$$
\begin{aligned}
R(u)_{i-\frac{1}{2}}=\frac{1}{\varepsilon^{(1)} F_{i}^{p}+\varepsilon^{(2)}\left(1-F_{i}^{p}\right)}\left[\varepsilon^{(1)}\left(a^{(1)}+b^{(1)} \sqrt{u_{i}^{2}+w_{c}^{2}}\right) F_{i}^{p}+\right. \\
\left.\varepsilon^{(2)}\left(a^{(2)}+b^{(2)} \sqrt{u_{i}^{2}+w_{c}^{2}}\right)\left(1-F_{i}^{p}\right)\right] u_{i}
\end{aligned}
$$

The friction force in the cell with index $i+1$ : 


$$
\begin{aligned}
R(u)_{i+\frac{1}{2}}=\frac{1}{\varepsilon^{(1)} F_{i+1}^{p}+\varepsilon^{(2)}\left(1-F_{i+1}^{p}\right)}[ & \varepsilon^{(1)}\left(a^{(1)}+b^{(1)} \sqrt{u_{i}^{2}+w_{c}^{2}}\right) F_{i+1}^{p}+ \\
& \left.\varepsilon^{(2)}\left(a^{(2)}+b^{(2)} \sqrt{u_{i}^{2}+w_{c}^{2}}\right)\left(1-F_{i+1}^{p}\right)\right] u_{i}
\end{aligned}
$$

in which $w_{\mathrm{c}}$ is approximated as the simple average of the vertical velocities at the position of $u_{\mathrm{i}}$ :

$$
w_{c}=\frac{1}{4}\left(w_{i, k}+w_{i, k-1}+w_{i+1, k}+w_{i+1, k-1}\right)
$$

Then, the total discretization of the friction force term becomes:

$$
\int_{\Omega} R(u) d V \approx F_{i}^{u} \Delta x_{i}^{p} \Delta z_{k}^{w} \frac{R_{i-\frac{1}{2}} \Delta x_{i}^{u}+R_{i+\frac{1}{2}} \Delta x_{i+1}^{u}}{\Delta x_{i}^{u}+\Delta x_{i+1}^{u}}
$$

\section{Time discretization of the momentum equation}

The space discretization of the continuity equation and the momentum equation can be written in matrix form and combined with a Forward Euler time integration:

$$
\begin{aligned}
& M_{\varepsilon} \mathbf{u}^{n+1}=0 \\
& \Omega_{\varepsilon} \frac{\mathbf{u}^{n+1}-\mathbf{u}^{n}}{\Delta t}=-C_{\varepsilon}\left(\mathbf{u}^{n}\right) \mathbf{u}^{n}+v D_{\varepsilon} \mathbf{u}^{n}-\frac{1}{\rho} G_{\varepsilon} \mathbf{p}^{n+1}+F_{\varepsilon}-\hat{R}_{\varepsilon} \mathbf{u}^{n+1}
\end{aligned}
$$

In (27), the subscripts $\varepsilon$ indicate that we have accounted for the porosity in the space discretization. Note that the convective term is non-linear, i.e. the coefficients of the matrix depend on the velocity $u$. Also note in this equation that the convective and viscous term are explicit and the pressure term and the friction force term are implicit.

We introduce an auxiliary vector field $\tilde{\mathbf{u}}^{n}$ :

$$
\tilde{\mathbf{u}}^{n}=\mathbf{u}^{n}-\Delta t \Omega_{\varepsilon}^{-1}\left(C_{\varepsilon}\left(\mathbf{u}^{n}\right) \mathbf{u}^{n}-v D_{\varepsilon} \mathbf{u}^{n}-F_{\varepsilon}\right)
$$

combine it with the momentum equation and rearrange terms to obtain:

$$
\mathbf{u}^{n+1}=\left[1+\Delta t \Omega_{\varepsilon}^{-1} \hat{R}_{\varepsilon}\right]^{-1}\left(\tilde{\mathbf{u}}^{n}-\frac{\Delta t}{\rho} \Omega_{\varepsilon}^{-1} G_{\varepsilon} \mathbf{p}^{n+1}\right)
$$

Eq. (29) is substituted into the discrete continuity equation. The result is a Poisson equation for the pressure that reads:

$$
M_{\varepsilon}\left[1+\Delta t \Omega_{\varepsilon}^{-1} \hat{R}_{\varepsilon}\right]^{-1} \Omega_{\varepsilon}^{-1} G_{\varepsilon} \mathbf{p}^{n+1}=\frac{\rho}{\Delta t} M_{\varepsilon}\left[1+\Delta t \Omega_{\varepsilon}^{-1} \hat{R}_{\varepsilon}\right]^{-1} \tilde{\mathbf{u}}^{n}
$$

From this equation the pressure is solved, after which the velocities at the new time level are found from (29).

\section{VERIFICATION STUDY}

To verify the implementation of the permeable flow extension in COMFLOW we compare numerical results to an analytical solution. The problem concerns wave interaction with a permeable structure. We will derive an analytical expression for the reflection coefficient and compare it to COMFLOW results.

Fig. 4 shows a permeable structure in waves. The structure is a rectangular block with porosity $\varepsilon$ that extends in vertical direction from the bottom to an elevation far beyond the free surface. In horizontal direction, the left boundary of the block is placed at a certain position that we will indicate by $x_{0}$. The right boundary is at positive infinity. 
Waves propagate from left to right through the open region; this is what we will call the incoming direction. When waves reach the structure, part of the wave energy will reflect back in opposite direction. The other part of the wave energy is transmitted through the structure, where it will dissipate as waves propagate further into the permeable region. The gradual decrease of wave energy will become apparent in a surface elevation that decreases with distance inside the structure.

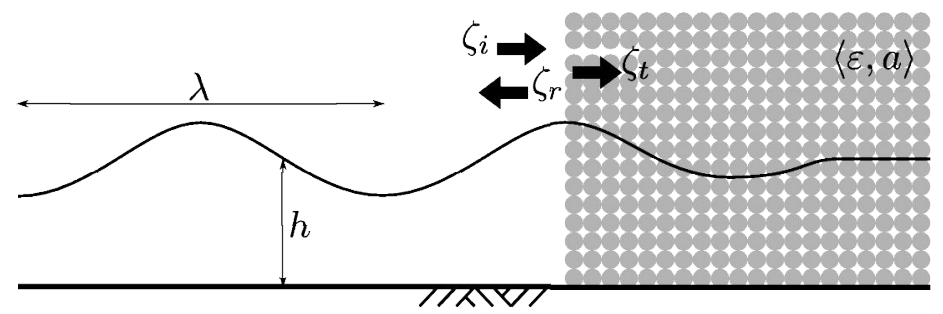

Figure 4. Situation sketch for the problem of wave reflection and transmission near the boundary of a permeable structure.

\section{Equations in the open region}

The wave length will be much longer than the water is deep, $\lambda>>h$, which allows us to employ the shallow water assumption. It will also be assumed that the amplitude of the waves is much smaller than one, $\zeta_{a} / h<<1$. With these assumptions, the Navier-Stokes equations for the conservation of mass and momentum reduce to the linear shallow water equations:

$$
\begin{aligned}
& \frac{\partial \zeta}{\partial t}+h \frac{\partial u}{\partial x}=0 \\
& \frac{\partial u}{\partial t}+g \frac{\partial \zeta}{\partial x}=0
\end{aligned}
$$

In these equations, $\zeta$ is the free surface elevation, $h$ is the water depth, $g$ the acceleration of gravity and $u$ the horizontal velocity.

The dispersion relation for this set of equations and waves with frequency $\omega$ and wave number $k$ equals:

$$
\omega^{2}=g h k^{2}
$$

With the dispersion relation, we can obtain the following expression for the horizontal velocity:

$$
u=\sqrt{\frac{g}{h} \zeta}
$$

\section{Equations in the permeable region}

When dealing with permeable structures, we need to account for the porosity and the resistance force inside the structure, which we model by means of a Darcy-type coefficient in combination with the velocity. The linear shallow water equations change as follows:

$$
\begin{aligned}
& \varepsilon \frac{\partial \zeta}{\partial t}+\frac{\partial(h \varepsilon u)}{\partial x}=0 \\
& \varepsilon \frac{\partial u}{\partial t}+g \varepsilon \frac{\partial \zeta}{\partial x}=-\varepsilon \hat{a} u
\end{aligned}
$$

in which $\hat{a}$ represents the Darcy coefficient.

For the set of equations in (34), the dispersion relation is as follows:

$$
\omega^{2}-i \hat{a} \omega=g h k^{2}
$$


With the dispersion relation, we can relate the surface elevation to the horizontal velocity inside the permeable structure:

$$
u=\sqrt{\frac{g}{h}} \sqrt{\frac{\omega^{2}}{\omega^{2}-i \hat{a} \omega} \zeta}
$$

\section{Transition conditions over the interface between regions}

Eq. (31) is used to solve the wave kinematics in the region outside the permeable structure; Eq. (34) for the kinematics inside the permeable structure. Neither of these equations, however, is valid at the boundary of the permeable structure. At the interface between the region with a porosity $\varepsilon=1$ and the region with porosity $\varepsilon \neq 1$, which is modeled as a discontinuity in porosity, we cannot resolve the derivative of $\varepsilon u$ in $x$-direction. At the interface, another set of equations is required. These equations will describe conservation of mass and conservation of energy.

\section{Mass conservation}

Mass conservation over the interface yields:

$$
h_{L} \varepsilon_{L} u_{L}=h_{R} \varepsilon_{R} u_{R}
$$

Here, variables left of the interface are denoted by the subscript $L$ and variables right of the interface are denoted by the subscript $R$.

\section{Energy conservation}

To obtain a similar expression as (37) for the conservation of energy, we first have to derive an energy equation. An energy equation is obtained when we multiply the first expression in Eq. (34) by $g \zeta$ and the second expression by $h u$ :

$$
\begin{gathered}
g \zeta \cdot\left[\varepsilon \frac{\partial \zeta}{\partial t}+\frac{\partial(h \varepsilon u)}{\partial x}=0\right] \\
h u \cdot\left[\frac{\partial(\varepsilon u)}{\partial t}+g \varepsilon \frac{\partial \zeta}{\partial x}=-\varepsilon \hat{a} u\right]
\end{gathered}
$$

and combine the two expressions into one relation that describes the change in total energy (potential and kinetic):

$$
\frac{\partial}{\partial t}\left(\frac{1}{2} h \varepsilon u^{2}+\frac{1}{2} g \varepsilon \zeta^{2}\right)+g \frac{\partial}{\partial x}(h \varepsilon u \zeta)=-h \varepsilon \hat{a} u^{2}
$$

From Eq. (39), we find that for energy to be conserved over a boundary of infinitesimal size, the following relation should apply:

$$
h_{L} \varepsilon_{L}(u \zeta)_{L}=h_{R} \varepsilon_{R}(u \zeta)_{R}
$$

in which variables to the left of the boundary are indicated with subscript $L$ and variables to the right of the boundary are indicated with subscript $R$.

If we now substitute the analytical expression for $u$ and $\zeta$ into the relations for mass conservation (37) and energy conservation (40), then we can derive an analytical expression for the reflection coefficient $K_{\mathrm{r}}$ and the transmission coefficient $K_{\mathrm{t}}$.

\section{Reflection and transmission coefficients}

At the interface, we need to relate three wave systems: left of the boundary we will have so-called incoming waves and reflected waves. At the right-hand side of the boundary we will have transmitted waves. These wave systems and their orientation are also shown in Fig. 4. 
Now consider the following relations, in which the total signal of the surface elevation and the velocity $u$ on either side of the boundary, are separated into the incoming, the reflected and the transmitted parts of the signal:

$$
\begin{aligned}
& \zeta_{L}=\zeta_{i}+\zeta_{r} ; \quad \zeta_{R}=\zeta_{t} \\
& u_{L}=u_{i}+u_{r} ; \quad u_{R}=u_{t} \\
& u_{i}=\sqrt{\frac{g}{h}} \zeta_{i} ; \quad u_{r}=-\sqrt{\frac{g}{h}} \zeta_{r} ; \quad u_{t}=\sqrt{\frac{g}{h}} \sqrt{\frac{\omega^{2}}{\omega^{2}-i \hat{a} \omega}} \zeta_{t}
\end{aligned}
$$

Here, the subscripts $i$ and $r$ indicate the incoming and reflected part of the wave signal, and the subscript $t$ indicates the transmitted part.

Substitution of these relations into the equations for mass and energy conservation yields:

$$
\begin{aligned}
& \sqrt{h_{L}} \varepsilon_{L}\left(\zeta_{i}-\zeta_{r}\right)=\sqrt{h_{R}} \varepsilon_{R} \sqrt{\frac{\omega^{2}}{\omega^{2}-i \hat{a} \omega}} \zeta_{t} \\
& \sqrt{h_{L}} \varepsilon_{L}\left[\left(\zeta_{i}+\zeta_{r}\right)\left(\zeta_{i}-\zeta_{r}\right)\right]=\sqrt{h_{R}} \varepsilon_{R} \sqrt{\frac{\omega^{2}}{\omega^{2}-i \hat{a} \omega} \zeta_{t}^{2}}
\end{aligned}
$$

From (42), we can derive that the reflection coefficient $\mathrm{Kr}$ equals:

$$
K_{r}=\frac{\zeta_{r}}{\zeta_{i}}=\left|\frac{1-\sqrt{\frac{h_{R}}{h_{L}}} \frac{\varepsilon_{R}}{\varepsilon_{L}} \sqrt{\frac{\omega^{2}}{\omega^{2}-i \hat{a} \omega}}}{1+\sqrt{\frac{h_{R}}{h_{L}}} \frac{\varepsilon_{R}}{\varepsilon_{L}} \sqrt{\frac{\omega^{2}}{\omega^{2}-i \hat{a} \omega}}}\right|
$$

The transmission coefficient then equals:

$$
K_{t}=\frac{\zeta_{t}}{\zeta_{i}}=1+K_{r}=\left|\frac{2}{1+\sqrt{\frac{h_{R}}{h_{L}} \frac{\varepsilon_{R}}{\varepsilon_{L}}} \sqrt{\frac{\omega^{2}}{\omega^{2}-i \hat{a} \omega}}}\right|
$$

\section{Numerical simulation}

A simulation for waves near a permeable structure is performed with COMFLOW. The computational domain extends from $x=0 m$ to $x=180 m$ in horizontal direction; in vertical direction the bottom of the domain is at $z=-1.05 \mathrm{~m}$ and the top of the domain at $\mathrm{z}=0.45 \mathrm{~m}$. The grid resolution in horizontal direction is $\Delta x=0.5 \mathrm{~m}$; in vertical direction $\Delta z=0.1 \mathrm{~m}$.

At $x_{0}=90 \mathrm{~m}$, the starting position of the structure is defined. The structure extends from the bottom of the domain to the top of the domain and from $x_{0}$ to the end of the domain at $x=180 \mathrm{~m}$. The porosity of the structure $\varepsilon=0.5$ and the Darcy-coefficient $\hat{a}=0.5 \mathrm{~s}^{-1}$.

The mean free surface is at $z=0$. As a result, the depth equals $h=1.05 \mathrm{~m}$. The wave period is $T=$ $10 \mathrm{~s}$ and the time step during the entire simulation was set at $\Delta t=10^{-3}$. The wave length at this period and depth is $\lambda=31 \mathrm{~m}$. The wave height is $H=10^{-3} \mathrm{~m}$.

At the inflow boundary at $x=0$, a Generating Absorbing Boundary Condition (GABC) was specified, see Wellens et al. (2009) and Borsboom et al. (2001). An integral part of the GABC is that the actual linear dispersion relation is approximated by means of a rational approximation in terms of the dimensionless wave number $k h$ : 


$$
c=\sqrt{g h} \sqrt{\frac{\tanh (k h)}{k h}} \approx \sqrt{g h} \frac{a_{0}+a_{1}(k h)^{2}}{1+b_{1}(k h)^{2}}
$$

in which $k$ is the wave number, $h$ is the water depth and $a_{0}, a_{1}$ and $b_{1}$ dimensionless coefficients that should be specified as input to COMFLOW.

With the GABC we prevent spurious re-reflection of waves back into the domain. The coefficients of the boundary condition were set to $a_{0}=0.99, a_{1}=0, b_{1}=0, k h=0.20$. These are the optimal settings for regular waves with this period.

The simulation was run for 300 seconds. There was ample time after a stationary situation was obtained; during this time, the reflection coefficient was determined by means of a least square method.

In Fig. 5, a snapshot of the simulation at $t=143 \mathrm{~s}$ with the surface elevation as a function of the position in the domain is shown. Here we can clearly see the decreasing surface elevation inside the permeable structure. The figure also shows that the mean free surface inside the permeable structure has been elevated with respect to $z=0$; this will not be investigated in this document.

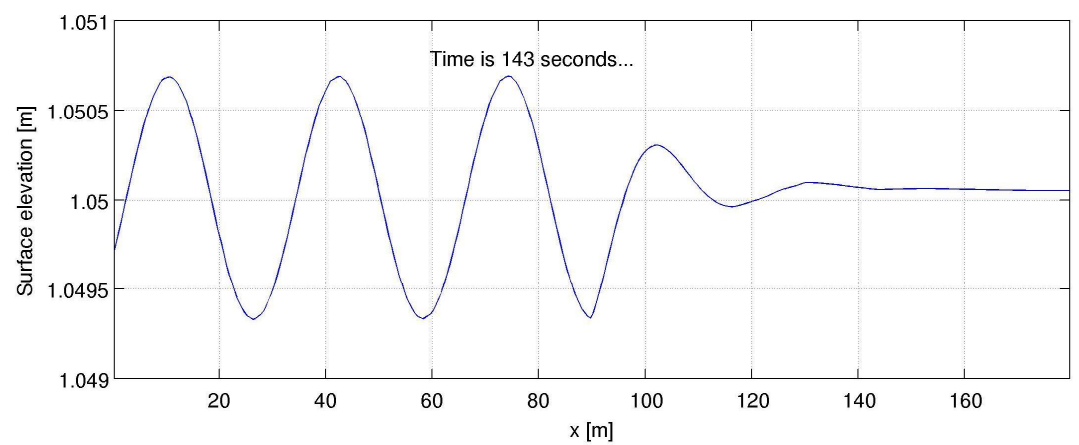

Figure 5. Snapshot of the simulation at $t=143 \mathrm{~s}$ : surface elevation in the domain with a permeable structure starting at $x=90 \mathrm{~m}$.

Table 1 shows the reflection coefficient that was determined from the simulation with a least-square analysis and the coefficient that was determined from Eq. (43). They compare reasonably well. The difference between the two may be explained by the following arguments:

- the analytical reflection coefficient was determined with the shallow water equations $(k h=0)$, whereas in the actual simulation there was some dispersion $(k h=0.20)$

- discretization errors are small, but may be of influence

- non-linearities, however small, may play a role

The dominant factor in the explanation of the difference is thought to be dispersion.

\begin{tabular}{|l|l|l|}
\hline \multicolumn{3}{|l|}{ Table 1. Reflection coefficients } \\
\hline Coefficient & Analytical & Simulation \\
\hline Reflection $K_{\mathrm{r}}$ & 0.42 & 0.45 \\
\hline
\end{tabular}

\section{VALIDATION}

Experimental results are used to validate the implementation of permeable flow in COMFLOW. We will only consider regular waves. The permeable structure represents a breakwater with a core of relatively fine material and an armour layer with relatively coarse rock. The outer measures of the structure are shown in Fig. 6. The base of the structure is about 3 meters wide, the top of the structure $0.35 \mathrm{~m}$ wide at an elevation of $0.93 \mathrm{~m}$. The slopes are $1: 1.5$. The mean free surface in the experiments was at an elevation of $0.60 \mathrm{~m}$ 


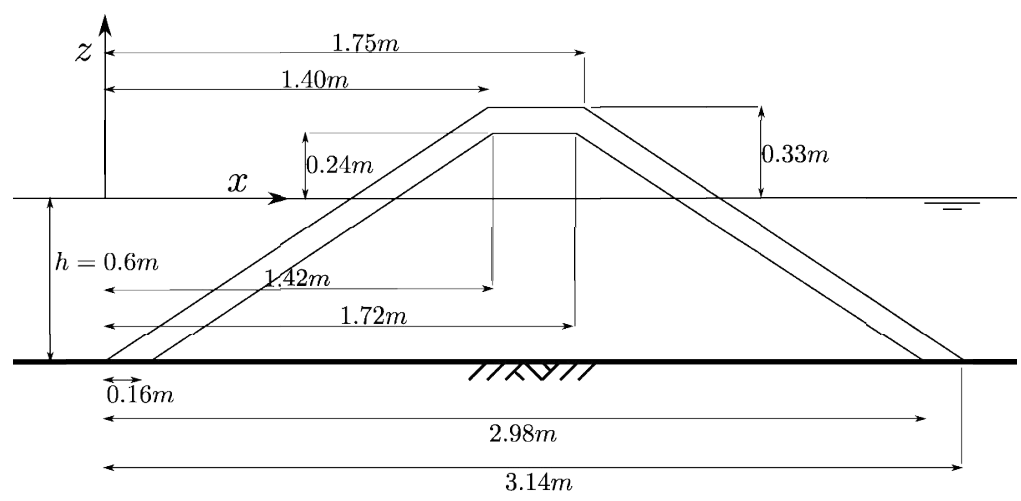

Figure 6. Outer measures of the permeable structure. The breakwater consists of an armour layer and a core.

The positions of the pressure sensors and the wave gauges are shown in Fig. 7. In vertical direction the pressure sensors are $0.060 \mathrm{~m}$ apart, in horizontal direction the sensors are between $0.090 \mathrm{~m}$ and $0.27 \mathrm{~m}$ apart. The highest pressure sensors are $0.060 \mathrm{~m}$ below the mean free surface. The four leftmost wave gauges are $0.12 \mathrm{~m}$ apart; the distance between the two rightmost wave gauges is $0.75 m$.

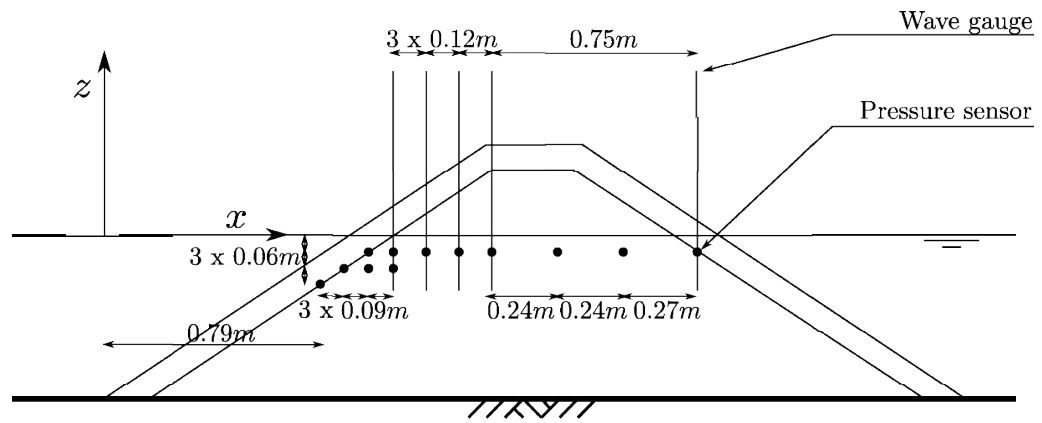

Figure 7. Sensor locations in the experiment. Wave gauges are represented as vertical lines; pressure sensors are shown as dots.

Figs. 6 and 7 are schematized representations of the actual experimental setup. Fig. 8 shows a composition of photos that were taken during construction. It shows the position of the wave gauges and the pressure sensors in the right part of the figure. The mount for the pressure sensors is shown on the left. The centre part of the figure shows the complete structure after construction.

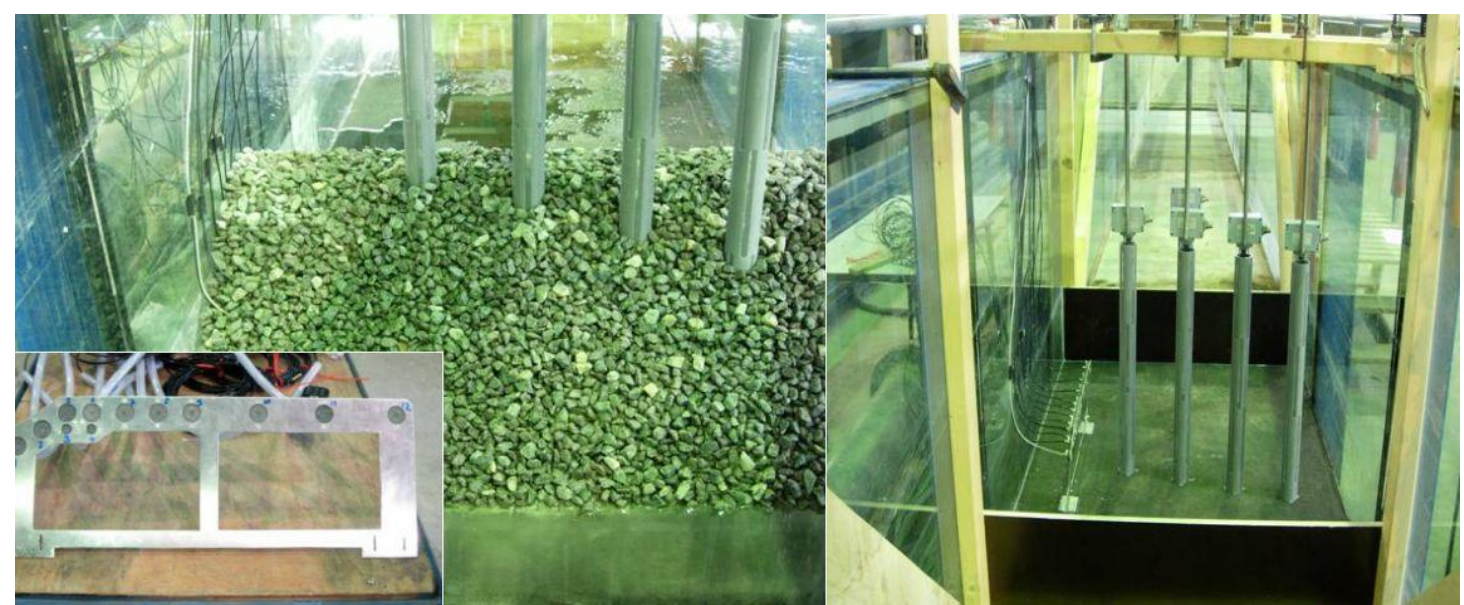

Figure 8. Experimental setup in the flume. The mount with pressure sensors is shown on the left; the array with wave gauges is shown on the right; the centre image shows the complete structure. 
The porosity of the different layers was carefully measured in the experimental setup itself. In Table 2, the porosities of the layers are listed, as well as the $D_{\mathrm{n} 50}$ of the material in the layers.

\begin{tabular}{|l|l|l|}
\hline \multicolumn{3}{|l|}{ Table 2. Porosity and nominal diameter } \\
\hline Layer & Porosity [-] & $D_{\mathrm{n} 50}[\mathrm{~m}]$ \\
\hline Core & 0.44 & 0.016 \\
\hline Armour & 0.44 & 0.038 \\
\hline
\end{tabular}

In this article, two regular wave tests will be considered. These tests are identified by brla and br7a. Table 3 shows the wave heights and periods that were specified during these tests.

\begin{tabular}{|l|l|l|l|}
\hline \multicolumn{4}{|l|}{ Table 3. Test programme } \\
\hline Test & $\mathrm{H}[\mathrm{m}]$ & $\mathrm{T}[\mathrm{s}]$ & $\lambda[\mathrm{m}]$ \\
\hline br1a & 0.060 & 1.6 & 3.27 \\
\hline br7a & 0.075 & 1.3 & 2.42 \\
\hline
\end{tabular}

\section{Numerical simulations}

The numerical simulations are performed with COMFLOW. The computational domain is $2 \mathrm{D}$ and extends from $x=-5.0$ to $x=5.0 m$ in horizontal direction and from $z=-0.60$ to $z=0.4 m$ in vertical direction. The number of cells in $x$-direction is 400 ; hence, the grid size $\Delta x$ is $0.025 \mathrm{~m}$. The same grid size was used in vertical direction. With this resolution, there are about 6 cells in the armour layer and there are at least 100 cells in the wave length.

In the simulations, the structural geometry was specified with the same dimensions and porosities as in the experiment. Recall that the (turbulent) interaction of the flow with the permeable structure is modeled with a resistance force that is a function of the velocity:

$$
R=(\hat{a}+\hat{b}|u|) u
$$

In Van Gent (1995), expressions are given that relate the Darcy coefficient and the Forchheimer coefficient to the porosity $\varepsilon$ and the average unit diameter of the material. The expression for $a$ is:

$$
a=\alpha \frac{(1-\varepsilon)^{2}}{\varepsilon^{3}} \frac{v}{g d_{n 50}^{2}}
$$

in which Greek letter $\alpha$ is a dimensionless coefficient that is determined from experiments and $v$ is the viscosity of water. Because the scaling of the equations in Van Gent (1995) is different from the scaling used in COMFLOW, the Darcy type coefficient $a$ in Van Gent is related to $\hat{a}$ in ComFLOW in the following way:

$$
\hat{a}=g \varepsilon a
$$

The expression for the Forchheimer coefficient in Van Gent (1995) reads:

$$
b=\beta \frac{1-\varepsilon}{\varepsilon^{3}} \frac{1}{g d_{n 50}}
$$

In (48), $\beta$ is a dimensionless coefficient found from experiments. Again, because the scaling in COMFLOW is different, the following equation relates $b$ to $\hat{b}$ :

$$
\hat{b}=g \varepsilon^{2} b
$$

The (dimensional) coefficients $\hat{a}$ and $\hat{b}$ have to be specified per layer. Table 4 shows the values for the coefficients that are used to represent the armour layer and the core in COMFLOW. 


\begin{tabular}{|l|l|l|l|l|}
\hline \multicolumn{5}{|l|}{ Table 4. Friction force coefficients } \\
\hline Test & $\alpha[-]$ & $\beta[-]$ & $\hat{a}\left[\mathrm{~s}^{-1}\right]$ & $\hat{b}\left[\mathrm{~m}^{-1}\right]$ \\
\hline Armour & 1100 & 0.3 & 1.14 & 9.65 \\
\hline Core & 1100 & 0.3 & 6.96 & 23.9 \\
\hline
\end{tabular}

The value for $\alpha$ is recommended by Van Gent (1995). The value for the coefficient $\beta$ is within the range of values that were obtained in an experiment by Van Gent-they were between 0.3 and 1.1.

The wave kinematics at the inflow end of the computational domain are determined by means of Rienecker-Fenton theory, see Rienecker and Fenton (1981). At this end of the domain, a Generating Absorbing Boundary Condition (GABC) is specified, see Wellens et al. (2009).

The GABC sends in waves in positive $x$-direction, while preventing re-reflection of waves propagating in negative $x$-direction that originate from the interaction with the structure. At the other end of the domain, the same boundary condition is specified without incoming waves. The coefficients for the boundary condition in both simulations are shown in Table 5. They are the optimal settings for regular waves with the periods in the experiments.

\begin{tabular}{|l|l|l|l|l|}
\hline \multicolumn{6}{|l|}{ Table 5. GABC coefficients } \\
\hline Test & $a_{0}[-]$ & $a_{1}[-]$ & $b_{1}[-]$ & $k h[-]$ \\
\hline br1a & 0.84 & 0.0 & 0.0 & 1.15 \\
\hline br2a & 0.76 & 0.0 & 0.0 & 1.56 \\
\hline
\end{tabular}

The time step varied during the simulation, but it was never larger than $2.5 \cdot 10^{-3} \mathrm{~s}$. The total simulation time was 100 seconds. During this time, output was collected from the sensor locations specified in Figure 7; the output of the numerical simulations can therefore be compared directly to the output of the experiments.

The output of the numerical simulations are post-processed. After sufficient time, a stationary situation is obtained, in which the wave height at an $x$-coordinate no longer changes. This wave height is determined as the maximum free surface excursion minus the minimum free surface excursion at that location. The wave heights for five consecutive wave gauges inside the permeable structure are plotted in Fig. 9 for experiment br1a and br2a. From these figures we find that the COMFLOW results for the wave heights agree well with the experiments and that the dimensionless coefficients $\alpha$ and $\beta$ are independent of the wave parameters.
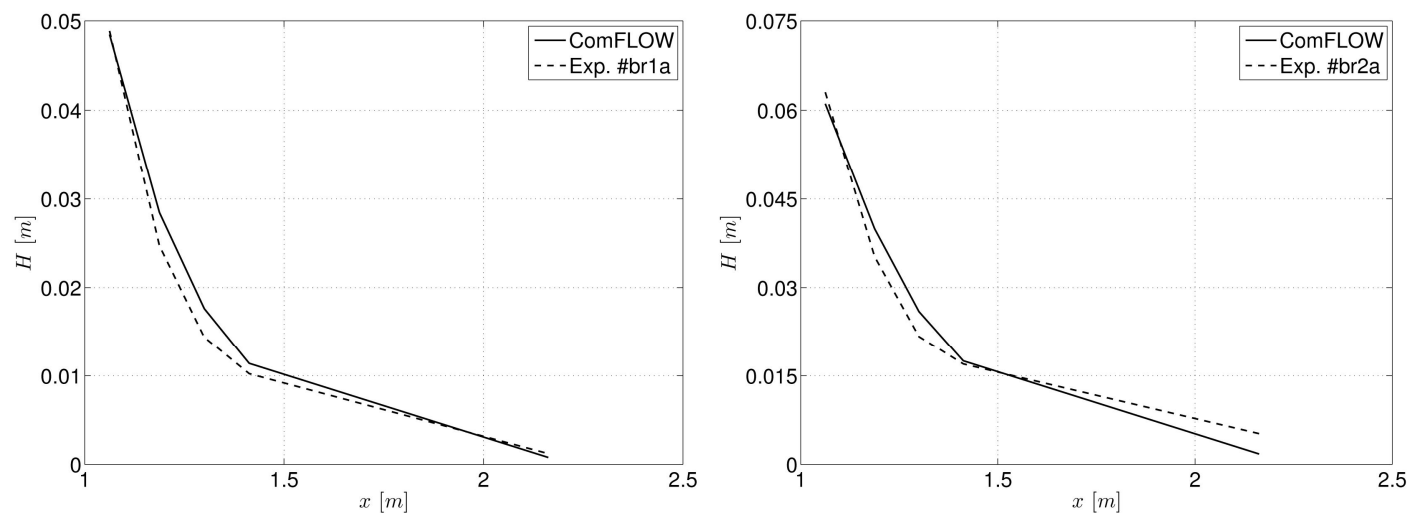

Figure 9. Wave heights inside the permeable structure.

The results for the pressure are also post-processed. When a stationary situation was obtained, the maximum pressure at a location was determined and the minimum pressure at that location was subtracted from the maximum to yield the equivalent of the wave height in terms of the pressure. Results for the top row of seven pressure sensors (see Fig. 7) are shown in Fig. 10. From these results, the conclusion is that also the pressures computed in CoMFLOW compare well to the experiments. 

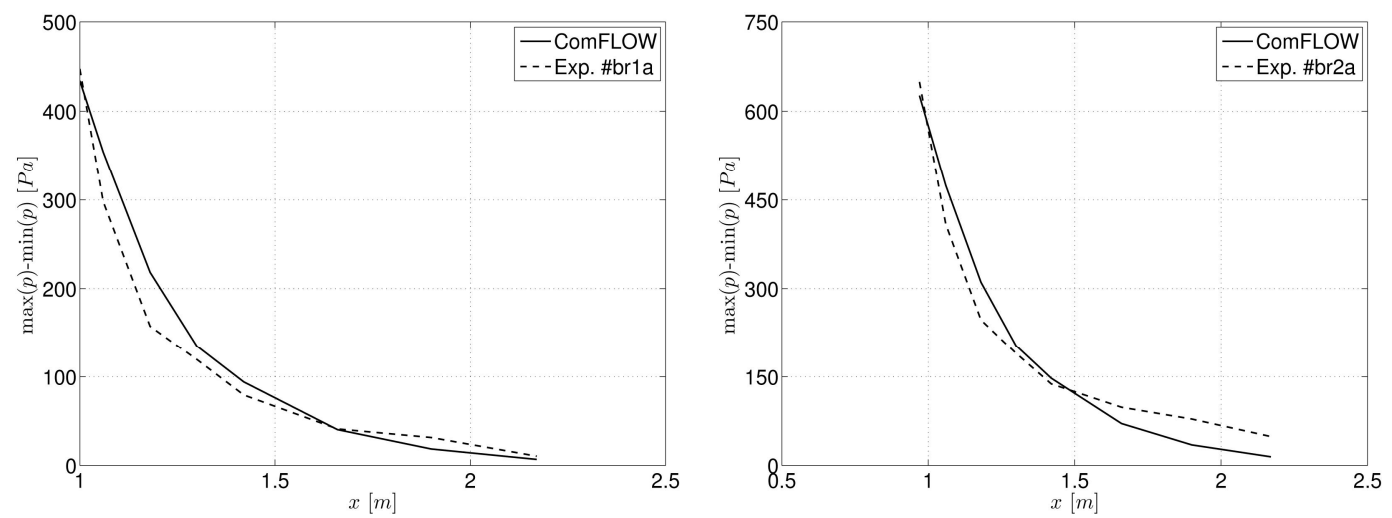

Figure 10. Pressures inside the permeable structure for experiment br1a and br2a.

\section{APPLICATIONS}

With the verification study and the validation, enough confidence was gained to apply the permeable flow implementation of COMFLOW in actual projects. One example is Van Gent et al. (2010). Another example is as follows: Deltares was asked to evaluate a preliminary design of an outfall for cooling water. The outfall is to be constructed near the coast: it consists of a weir some distance offshore from a dike. The weir is protected by two breakwaters, perpendicular to the orientation of the dike. The length of the breakwaters was determined by means of design rules, but it was uncertain whether these would apply in this situation. Therefore, the structure was modeled with COMFLOW.

The most extreme event is when the wave direction is in line with the breakwaters. With this event, the centre line of structure, in between breakwaters, acts as a symmetry plane. To save computational effort, only one breakwater, on one side of the symmetry plane is modeled. The composition of the breakwater, in the preliminary design stage, was unknown. It was estimated from experience that the porosity of the material would very likely be near 0.40 . It was also assumed that the entire breakwater is composed of one material with a rock diameter that is normally used in the armour layer of a structure. The permeability coefficients of the material are specified as $\hat{a}=0.02 \mathrm{~m}^{-1}$ and $\hat{b}=1.18 s^{-1}$. The dike is modeled as an impermeable structure.

At the inflow end of the domain, an irregular sea state was prescribed. According to the design rules, the significant wave height at the position of the weir should be reduced by $20 \%$. Fig. 11 shows a snapshot of the simulation. It shows the breakwater, the cross-section of the weir and the impermeable dike section. By means of the simulation, it was determined that for this sea state, the significant wave height near the weir was reduced with $31 \%$ with respect to the input spectrum.

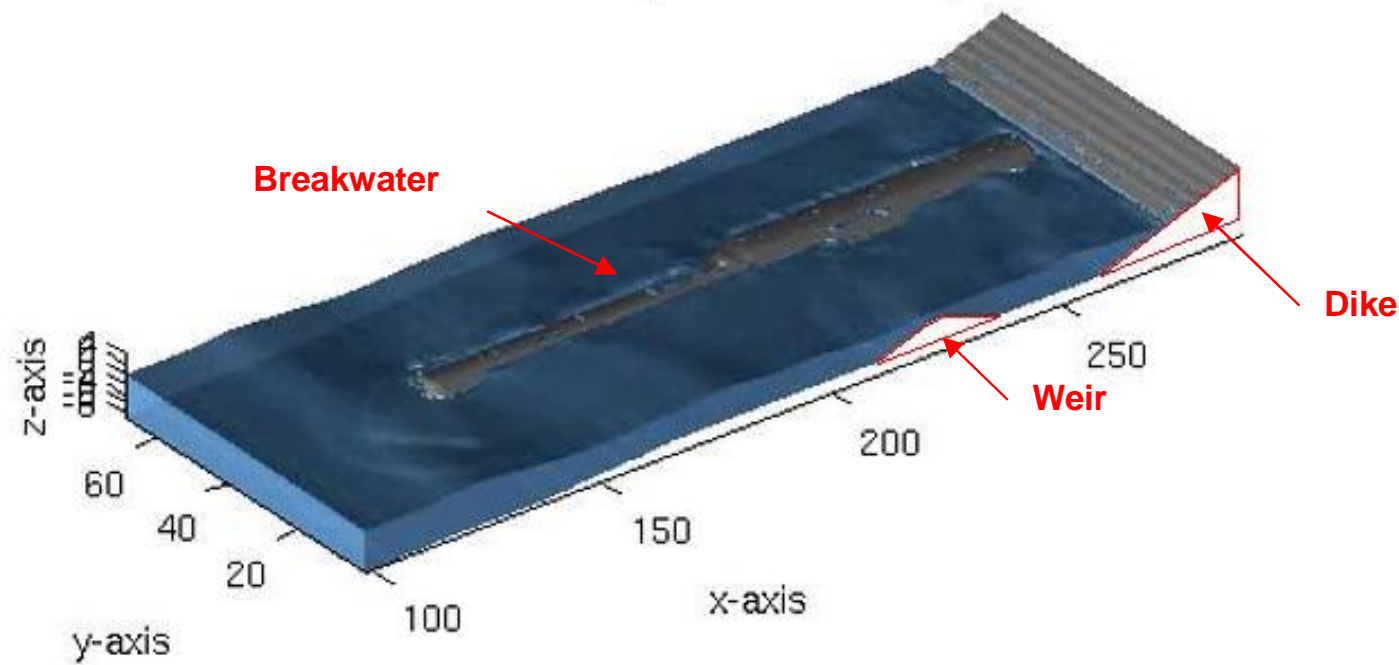

Figure 11. Snapshot of a 3D simulation with a weir protected by a breakwater. Wave direction is in line with the breakwater. 


\section{CONCLUSION}

COMFLOW has been extended with a permeable flow model. In the Navier-Stokes equations we account for the porosity and model the (turbulent) interaction of flow and structure with a friction force that depends on the flow velocity.

An analytical formulation for the reflection coefficient of a permeable structure has been determined by means of the shallow water equations. The analytical expression was compared to simulation results obtained with COMFLOW. The COMFLOW results compare reasonably well to the analytical expression.

COMFLOW simulations have been compared to experiments. The numerical results for pressure and surface elevation compare well to the measurements. It was found that with constant values for the material coefficients $\alpha$ and $\beta$, it is possible to represent a structure composed of layers of material with different nominal diameters in several sea states.

It must be noted that with the type of post-processing used in this study, we have only considered certain aspects of the physics. The aspects that were retained during post-processing were wave behavior and energy dissipation inside the permeable structure.

It is recommended to perform more validation. Irregular wave tests have also been performed with this structure and it is imperative that COMFLOW is validated with these tests. It is also recommended to perform more validation of COMFLOW with experiments in a $3 \mathrm{D}$ setup.

\section{ACKNOWLEDGEMENTS}

The authors would like to acknowledge Guido Wolters at Deltares for performing the experiments in this study. Bas Hofland at Deltares was project leader for the project with weir and breakwaters in the section concerning the applications. Ivo Wenneker was project leader for the project concerning the derivation, implementation and validation of the permeable flow equations in COMFLOW. And we are grateful to prof. Arthur Veldman of the University of Groningen for providing the CoMFLOW code, in which we could implement our research.

\section{REFERENCES}

Borsboom, M., J. Groeneweg, N. Doorn and M.R.A. van Gent (2001), Flexible Boundary Conditions for a Boussinesq Type Wave Model, Proceedings of Waves 2001, San Francisco

Kleefsman, K.M.T., G. Fekken, A.E.P. Veldman, B. Iwanowski, and B. Buchner (2005), A Volumeof-Fluid based simulation method for wave impact problems. Journal of Computational Physics, 206(1):363-393

Liu, P.L.F, P. Lin, K.A. Chang, and T. Sakakiyama (1999), Numerical Modeling of Wave Interaction with Porous Structures, Journal of Waterway, Port, Coastal and Ocean Engineering, 125, 322330

Rienecker, M.M. and J.D. Fenton (1981), A Fourier Approximation Method For Steady Water-Waves, Journal Of Fluid Mechanics, 104(MAR):119-137, 1981

Van Gent, M.R.A. (1995), Porous flow through rubble mound material, J. of Waterway, Port, Coastal and Ocean Engineering, Vol.121, no.3, pp.176-181, ASCE, New York

Van Gent, M.R.A. and I.M. van der Werf (2010), Stability of breakwater roundheads during construction, Proceedings of $32^{\text {nd }}$ International Conference on Coastal Engineering. Shanghai, China

Van Gent, M.R.A., P. Tönjes, H.A.H. Petit and P. van den Bosch (1994), Wave action on and in permeable structures, Proc. ICCE'94, Vol.2, pp.1739-1753, Kobe, Japan

Verstappen, R.W.C.P. and A. E. P. Veldman (2003), Symmetry-preserving discretization of turbulent flow. Journal of Computational Physics, 187(1):343-368.

Wellens, P.R., R. Luppes, A.E.P. Veldman and M.J.A. Borsboom (2009), CFD Simulations of a SemiSubmersible with Absorbing Boundary Conditions, Proceedings of OMAE2009, Honolulu

Wenneker, I., P.R.Wellens and R. Gervelas (2010), Volume-of-Fluid model ComFLOW simulations of wave impacts on a dike, Proceedings of $32^{\text {nd }}$ International Conference on Coastal Engineering. Shanghai, China 